PART II

THE STRUCTURE OF THE UNIVERSE

(Chairman: K. Rudnicki) 


\title{
SUPERCLUSTERING OF GALAXIES
}

\author{
G. O. A BELL \\ Dept. of Astronomy, University of California, Los Angeles, Calif., U.S.A.
}

\begin{abstract}
Evidence for superclustering of galaxies includes the local supercluster, the large deviation of the observed surface distribution of clusters of galaxies from a random one, the cell size dependence of the index of clumpiness, the results of covariance function tests for correlations between different cluster centers and between clusters and individual galaxies, and a power-spectrum analysis. All tests give results that indicate or are compatible with the existence of inhomogeneities in the distribution of matter in space with linear diameters of 50 to $100 \mathrm{Mpc}$. If these are dynamically stable superclusters, they probably have internal velocity dispersions in the range 1000 to $3000 \mathrm{~km} \mathrm{~s}^{-1}$. The possible effects of superclustering on dynamical studies of individual clusters are discussed briefly.
\end{abstract}

\section{Introduction}

The obvious tendency of galaxies to be located in clusters is more or less universally accepted by all observers. Hubble (1936), in fact, suggested that clustering may be a universal property of galaxies. Neyman and Scott, in collaboration with Shane and Swanson (Neyman and Scott, 1952; Neyman et al., 1953, 1954; Scott et al., 1954) have shown that the distribution of galaxies whose images are recorded by the Lick Astrographic Survey is compatible with the assumption that all galaxies are in clusters.

On the other hand, the possibility of the existence of larger units of matter has not been generally taken into account in the discussion of the large-scale distribution of matter in space, despite considerable observational evidence advanced to support that point of view. In fact, the existence of aggregates larger than clusters has been suspect and controversial. I think that this lack of serious consideration of what has often been called 'superclustering' has resulted, at least in large part, from the lack of a clear picture of what observers mean by superclustering.

For example, Zwicky (Zwicky, 1957, 1959; Zwicky and Rudnicki, 1963, 1966) has consistently rejected the hypothesis that there exist great globular clusters of clusters of galaxies, morphologically similar to, but on a larger hierarchy than ordinary galaxian clusters. Certainly no evidence has been advanced for such super clusters with hundreds or thousands of member clusters, each (like the Coma cluster) containing thousands of galaxies. The discussion of the nonexistence of these features has obscured the question of whether, in fact, those systems generally regarded as firstorder clusters really represent the largest inhomogeneities observed in the Universe.

Clusters of galaxies range from poor aggregates like the Local Group with only about 20 or so recognized or suspected members to systems like the Coma cluster with at least $10^{3}$ members brighter than $m_{v}=18.5$, and possibly tens of thousands of galaxies as massive as those least conspicuous members of the Local Group. Yet, the great clusters and small groups are not enormously different in linear diameter. A definitive radius has not yet been defined for even one cluster of galaxies, but at least 
within a factor of 3 to 5 the Coma cluster, the Local Group, and all other clusters are comparable, having diameters in the range 2 to $10 \mathrm{Mpc}$ (a Hubble constant, $H=$ $=50 \mathrm{~km} \mathrm{~s}^{-1} \mathrm{Mpc}^{-1}$ is assumed in this review).

A very large body of observational data, summarized below, suggests, however, that much larger aggregates of matter exist as inhomogeneities in the Universe. To put it another way, we cannot represent the observed distribution of matter in space with a model of a uniform or random distribution of non-interacting units, the largest of which have linear diameters of the order 5 to $10 \mathrm{Mpc}$. Strong spatial correlations exist between the locations of the centers of rich clusters, between cluster centers and individual galaxies, and between galaxies themselves, that extend over distances roughly an order of magnitude greater.

The scale of the largest inhomogeneities in the distribution of matter is clearly a datum of great cosmological importance, and it is to this question that our attention is directed here. Whereas some theoretical considerations (e.g., Peebles, 1973a) suggest that superclusters should be dynamical units, observational evidence for gravitational interactions between clusters is meager at best. On the other hand, we shall comment (in Section 4) on the influence of superclustering on the interpretation of data pertinent to dynamical studies of individual clusters.

\section{The Local Supercluster}

A local inhomogeneity in the distribution of groups and clusters has been suspected for several decades. Holmberg (1937) analyzed the distribution of double and multiple galaxies and presented evidence for a metagalactic cloud with a diameter (according to the Hubble constant adopted in this review) of the order $100 \mathrm{Mpc}$. Holmberg found the center of this cloud to lie in the general direction of the north galactic pole and at a distance of about $20 \mathrm{Mpc}$. Later, from his study of the distributions in direction and magnitude of some 4000 galaxies in the region of the north galactic pole, Reiz (1941) qualitatively confirmed Holmberg's conclusion.

More than a decade later de Vaucouleur $(1953,1956,1958)$ revived the idea of a local supercluster of galaxies. He finds the supercluster to have a diameter of about $75 \mathrm{Mpc}$ (for $H=50 \mathrm{~km} \mathrm{~s}^{-1} \mathrm{Mpc}^{-1}$ ), and to comprise the Local Group, the Virgo cluster, the Ursa Major cloud, and numerous other smaller groups and clusters. According to de Vaucouleur the system is flattened, so that the brighter galaxies concentrate toward a great circle in the sky (the supergalactic equator) with its pole at $l=47^{\circ}, b=5^{\circ}$. He believes that the center of the system lies in or near the Virgo cluster.

Further evidence for the reality of a local supercluster is provided by a study of Carpenter (1961; Abell, 1961), who investigated the distribution of images of galaxies on the Palomar Atlas prints in magnitude and direction. Carpenter found that galaxies with $m_{p g} \leqslant 13.5$ are highly concentrated along a $90^{\circ}$ sector of an $18^{\circ}$ strip centered on de Vaucouleurs' supergalactic equator. He finds a significantly smaller number of bright galaxies in adjacent $18^{\circ}$ strips straddling the supergalactic equator. In all three strips the numbers of galaxies of increasingly fainter magnitudes increase 
rapidly until $m_{p g}=13.5$ is reached, after which the numbers increase less rapidly. For $m_{p g} \geqslant 14.5$, however, the logarithm of the number of galaxies brighter than $m_{p g}$ increases roughly as $0.6 m_{p g}$ as would be expected if most of the fainter galaxies were remote ones beyond the limits of a local supercluster (see Figure 1).

De Vaucouleurs has also analyzed the radial velocities of galaxies along the supergalactic equator, and on the basis of this study he suggests that the supercluster is rotating and expanding. A significantly flattened, axially symmetrical supersystem of galaxies would, indeed, suggest rotation. It is the writer's opinion, however, that hard evidence for symmetry is at best weak. Moreover, at the rotation rate suggested by de Vaucouleurs, the present-day period of revolution of our Galaxy about its center would be of the order $10^{11} \mathrm{yr}$, and even though the system may be expanding it is doubtful that in the usually-assumed age of $10^{10} \mathrm{yr}$ radial symmetry and dynamical flattening could have been achieved. The indication of rotation, therefore, may be spurious. Nevertheless, the evidence of a local inhomogeneity in the distribution of galaxies with a diameter of the order $100 \mathrm{Mpc}$ is strong enough that we should seriously consider the possibility that it is a manifestation of a general second-order clustering.

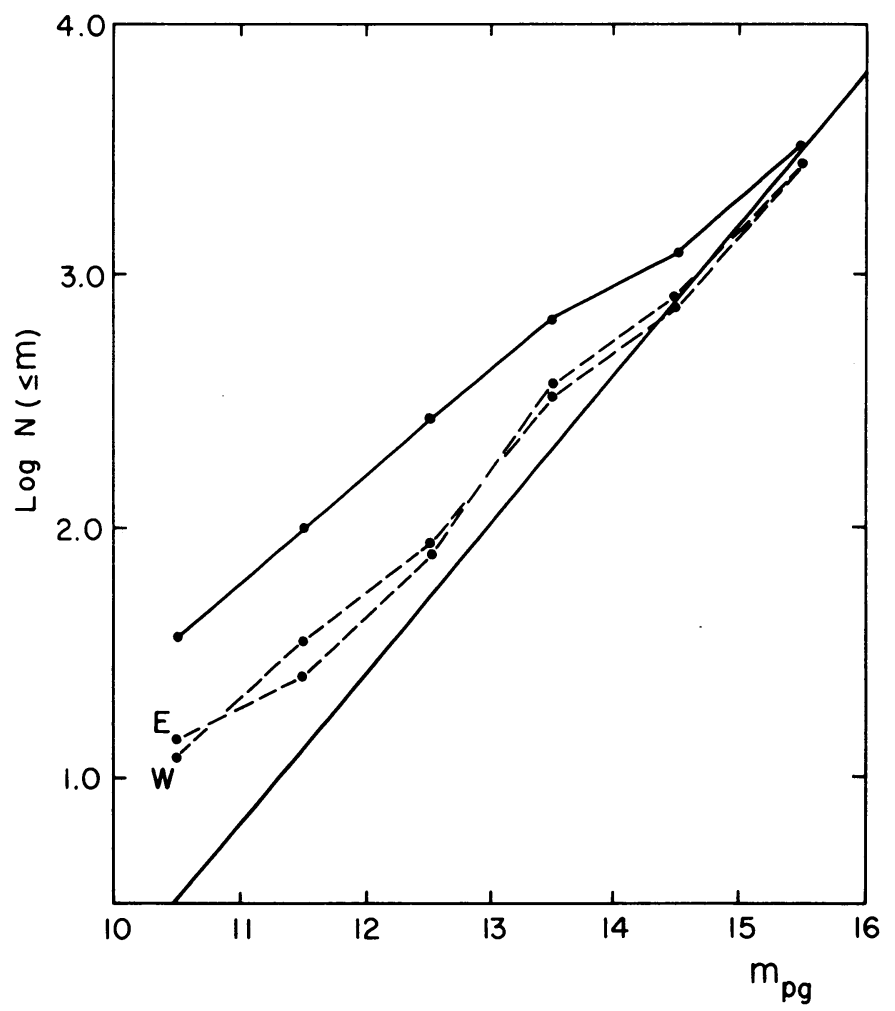

Fig. 1. Number of galaxies brighter than photographic magnitude $m_{p g}$. Solid line: galaxies in the strip along the supergalactic equator; dotted lines: galaxies in strips adjacent to the supergalactic equator. The heavy straight line has a slope of 0.6 (adapted from Carpenter). 


\section{Evidence for General Superclustering}

The distribution of rich clusters of galaxies in a homogeneous sample selected from the writer's catalogue (Abell, 1958) suggested a strong tendency for clusters to clump into larger groups (Figure 2). In fact, a $\chi^{2}$ test showed that the probability $P\left(\chi^{2}\right)$ of the frequency distribution, $N(t)$, of equal-area cells in the sky that contain $t$ clusters each being a random selection from a binomial distribution, $B(t)$, is only $10^{-61}$. Other effects besides second-order clustering could account for the non-random surface distribution of clusters - for example, interstellar and possible intergalactic obscuration, a large-scale anisotropy of the cluster distribution, and the incomplete identification of clusters behind the richer star fields near the Milky Way.

A case for superclustering was made, however, by comparing the observed and binomial frequency distributions separately for clusters in different distance classes, and for different cell areas. For clusters in each distance class $N(t)$ approaches $B(t)$ as the cell areas approach zero, for then every cell contains either one cluster or none. With increasing cell size, $N(t)$ departs more and more from $B(t)$, with $P\left(\chi^{2}\right)$ passing through a minimum, which for the more distant clusters (for which the sample is largest) is as low as $10^{-30}$ to $10^{-40}$. At still larger cell areas $P\left(\chi^{2}\right)$ increases again, mainly because the sample size diminishes (fewer large area cells fit into the sky than small area ones) and the deviation of $N(t)$ from $B(t)$ is less significant. $P\left(\chi^{2}\right)$ should also eventually increase with cell size if the cells become large compared to any anisotropies in the cluster distribution - that is, if superclustering is 'smoothed out'. Abell (1958, 1961) originally interpreted an observed inverse correlation between the cell diameters for which $P\left(\chi^{2}\right)$ is a minimum with the cluster distance class as an indication that the second-order clustering occurs on the same scale at all distances surveyed. This interpretation is not strictly justified because of the smaller significance of the results for large cell sizes. However, at cell sizes smaller than those for which $P\left(\chi^{2}\right)$ is at a minimum, the descent of $P\left(\chi^{2}\right)$ with cell size is steepest for the most distant clusters and least steep for the nearest, as one would expect for superclusters of a common scale displaying smaller angular sizes at greater distances.

The evidence that second-order clusters may have similar linear sizes at different distances argues against their being illusions produced by interstellar or intergalactic obscuration. Simple inspection of Figure 2 would also seem to rule out absorption as the cause of the clumpy cluster distribution; if apparent clumps of relatively near clusters are merely parts of a uniform or random distribution of clusters seen through holes in absorbing material, then apparent clumps of more remote clusters should be seen in the same directions, but certainly not between them, as is the case.

The distribution of clusters in Volumes 1, 2, 3, and 5 of the Catalogue of Galaxies and Clusters of Galaxies (Zwicky et al., 1961-68), the first four volumes of the catalogue to be published, have been analyzed in the same way with nearly identical results by Abell and Seligman $(1965,1967)$. Figure 3 displays some of these results in the form of separate plots of $\log P\left(\chi^{2}\right)$ as a function of cell diameter for the clusters Zwicky classifies as near, medium distant, distant, very distant, and extremely distant. To 


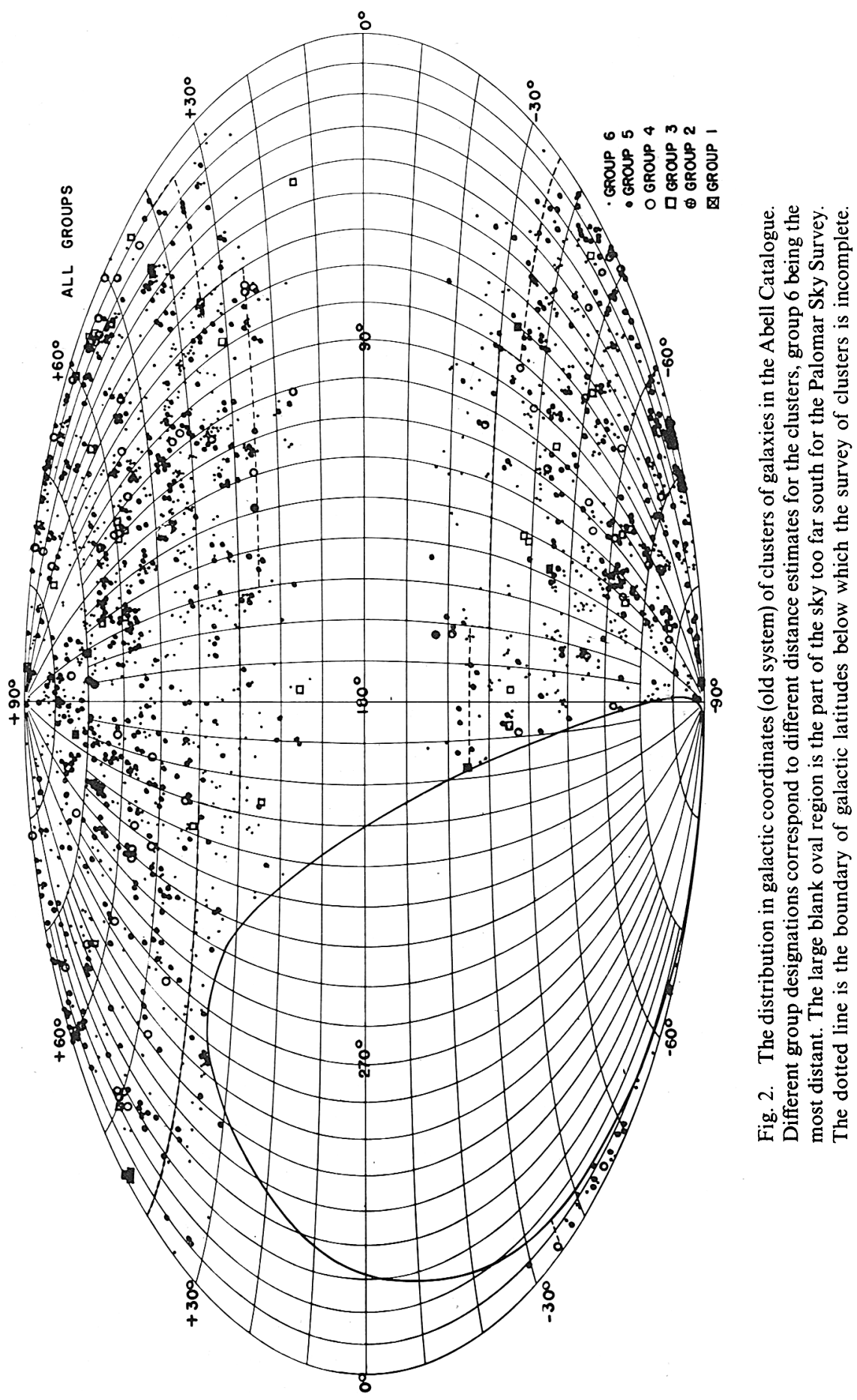




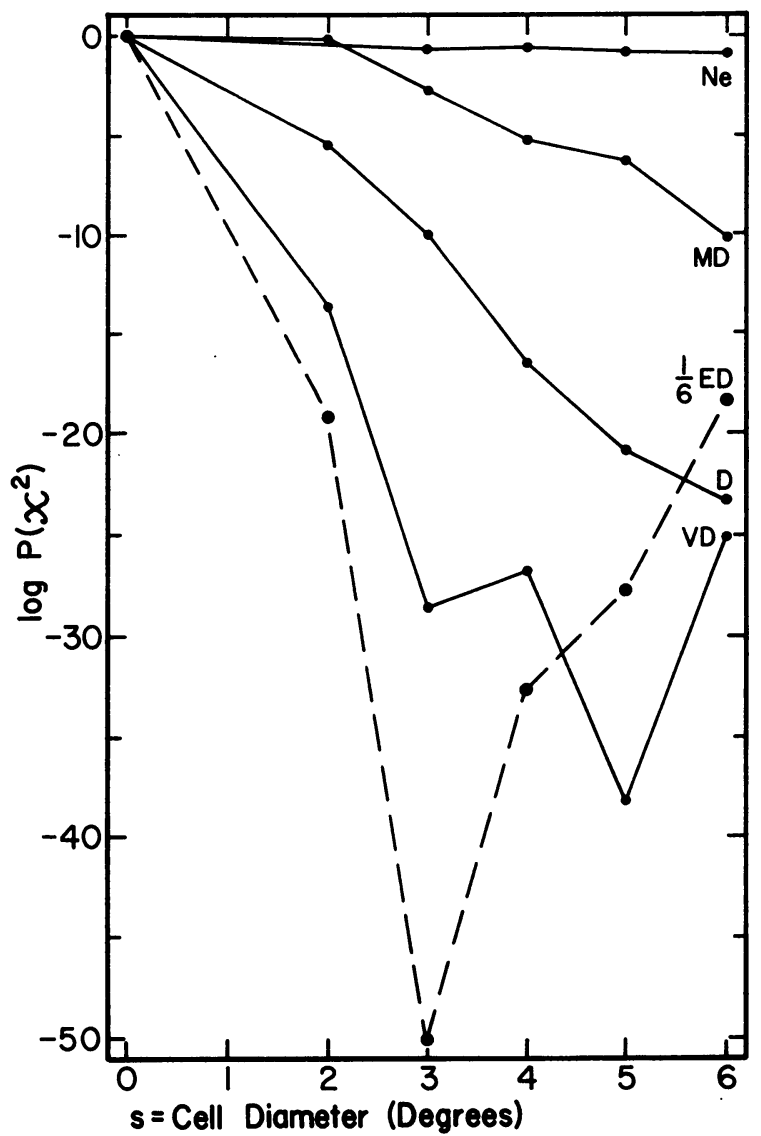

Fig. 3. The logarithms of the probability, $P\left(\chi^{2}\right)$, that larger values of $\chi^{2}$ would be obtained from frequency distributions of random samplings from a binomial distribution than were actually found from the frequency distributions of clusters in the Zwicky et al. (1961-68) Catalogue of Galaxies and Clusters of Galaxies, as functions of cell size. The five curves are for clusters Zwicky classifies as near (Ne), medium distant (MD), distant (D), very distant (VD), and extremely distant (ED). The latter logarithms of probabilities have been divided by 6 in order to show them on the same plot.

minimize the effects of the incompleteness of the Catalogue at low galactic latitudes, only clusters with $|b|>30^{\circ}$ were used in the calculation, except for the 'near' clusters, for which the entire Catalogue was used because of the small sample. Note that the values of $\log P\left(\chi^{2}\right)$ for extremely distant clusters were all divided by 6 in order to show them on the same plot.

Zwicky $(1953,1957)$ has studied the quantity $k(z, n)$, defined by

$$
k(z, n)=\frac{S_{1}}{S_{0}}
$$

where $S_{1}^{2}$ is the sample variance of the observed distribution of $n$ objects distributed among $z$ cells in a given solid angle in the sky, and $S_{0}^{2}$ is the variance expected if the 
$n$ objects are distributed randomly among the $z$ cells. When applied to the distribution of galaxies, $k(z, n)$ increases with decreasing $z$ (that is, with increasing cell size). Zwicky interpreted this result to be evidence for both clustering of galaxies and for intergalactic absorption. On the other hand, Neyman et al. (1954) investigated an analogous quantity, which they call the index of clumpiness, $K$, defined by

$$
K=\frac{\sigma_{1}}{\sigma_{0}}
$$

where $\sigma_{1}^{2}$ is the true variance of a theoretical distribution of $n$ objects among $z$ cells, computed on the assumption of no intervening absorption, and also on the assumption of complete clustering of the objects; $\sigma_{0}^{2}$ is the variance of the same $n$ objects distributed randomly. Neyman et al. (1954) show that with the given assumptions $K$ is a nondecreasing function of cell size. Thus Zwicky's result is compatible with complete clustering of galaxies, but in itself gives no information about intergalactic obscuration

Abell (1958) computed $k(n, z)$ as a function of cell size, $s$, for the clusters of galaxies in the Abell catalogue. For clusters of all distance groups, $k$ is a nondecreasing function of $s$, although the results are less significant than in the distribution of individual galaxies. Abell and Seligman (partly reported in Abell and Seligman, 1967) did a similar analysis of the larger sample of clusters in Volumes 1, 2, 3, and 5 of the Zwicky et al. (1961-68) catalogue. Figure 4 shows $k$ vs $s$ for all of the clusters in each of Zwicky's

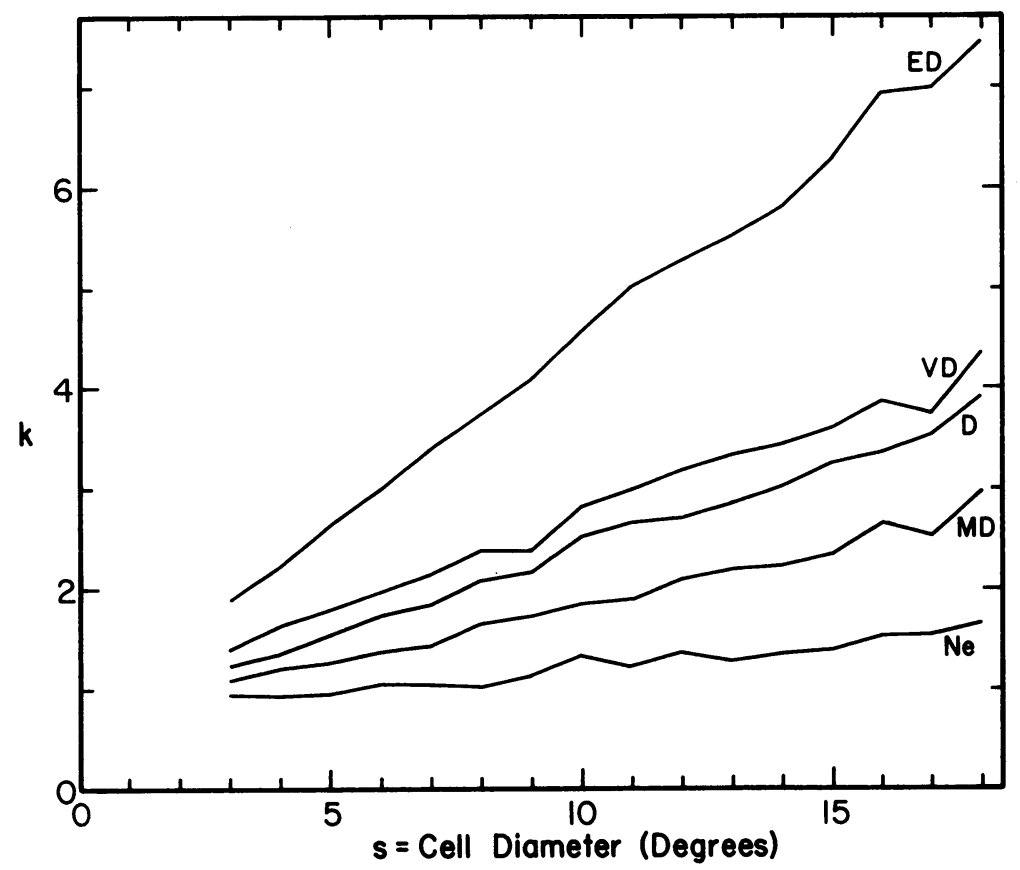

Fig. 4. The index of clumpiness, $k$, as a function of cell size for the clusters in Volumes 1, 2, 3 and 5 of the Zwicky et al. (1961-68) Catalogue for each of Zwicky's five distance categories. 
distance catagories in the four catalogue volumes. While not proving that superclustering exists, the behavior of $k$ is nevertheless that expected if all clusters are in second-order clusters.

Zwicky and Rudnicki (1966) and Karpowicz (1967) made similar analyses of the distributions of clusters in Volume 1 and Volume 2, respectively, of the Catalogue. They each obtained results similar to those of Abell and Seligman, but because of the smaller sample of clusters in any one catalogue volume their $k$ values are systematically smaller. These authors argue that the small values of $k$ are evidence against the clustering of clusters, but in fact the reverse is true. The values found by Zwicky and Rudnicki (1966) (up to $k=3$ ) and by Karpowicz (up to $k=6$ ) are very significant evidence for a nonrandom cluster distribution. The square of $k$ or $k^{-1}$ (whichever is greater than unity) is the well-known statistic $F$, widely used to test the compatibility of two different dispersions. From the $F$ distribution Abell and Seligman (1967) computed the probability of obtaining the values of $k$ displayed in Figure 4. As illustration, these probabilities are shown for the distant, very distant, and extremely distant clusters in Figure 5. (The large values of $F$ or $k$ for large $s$ are less significant than the smaller values at small $s$ because of the smaller number of degrees of freedom - that is, number of cells.) The probabilities shown in Figure 5 do not give information different from that of the $\chi^{2}$ probabilities, but the increase in $k$ with $s$ is interesting because the same pattern is predicted by general second-order clustering.

Numerous other investigators have attempted analyses of the distribution of rich clusters of galaxies in the published catalogues. Among them, Kiang and Saslaw (1969) computed serial correlations of Abell clusters in $50 \mathrm{Mpc}$ cubic cells to determine the three-dimensional cluster distribution, and find correlations over a scale of at least $100 \mathrm{Mpc}$ and possibly to $200 \mathrm{Mpc}$. Bogart and Wagoner (1973) performed nearest neighbor tests on the Abell clusters, and found that the distribution of nearest neighbor distances from half of the clusters (sources) to the other half (objects) has a significantly smaller mean than does the corresponding distribution when a set of random points is used for sources, indicating that the clusters are significantly clustered. Bogart and Wagoner estimated the scale of the clustering by rotating the 'object' half of the clusters in galactic longitude until the distribution of nearest neighbor distances approached the random one. The angular scale found for distance group 5 clusters is slightly greater than for the more distant group 6 clusters, suggesting a physical association of clusters; the corresponding linear scale is $\sim 200 \mathrm{Mpc}$.

The writer (Abell, 1957) attempted to calculate serial correlations for the positions of clusters in his catalogue in distance groups 5 and 6, and found weak evidence for correlation over angular distances of $5^{\circ}$ to $10^{\circ}$, but because of the limited computing facilities available to him at that time, the estimates of the correlation coefficients were rather rough. Abell and Seligman (1967), with somewhat improved computing techniques, performed a similar analysis of the Zwicky clusters, and found weak evidence for correlation over scales of up to $5^{\circ}$ for the very distant clusters.

Statistical analyses of three catalogues of extragalactic objects have been carried out recently by the Princeton group (Peebles, 1973b; Hauser and Peebles, 1973; 


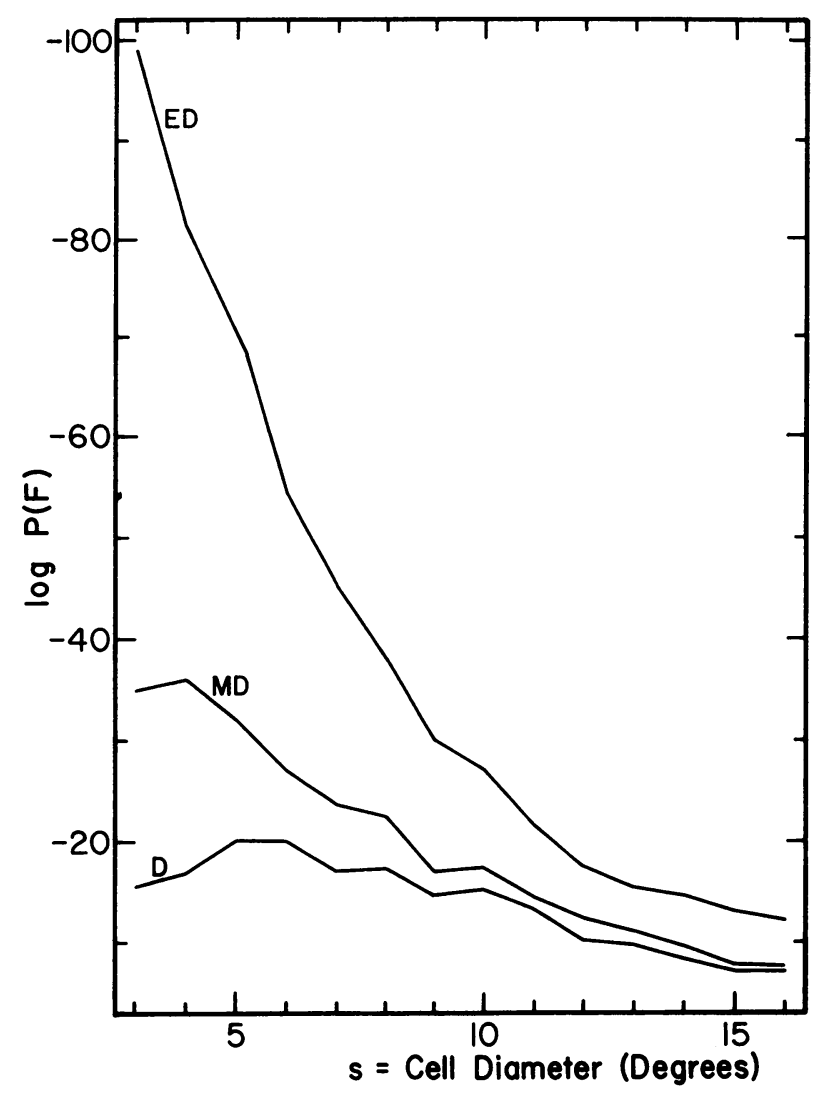

Fig. 5. The probabilities of obtaining larger $k$ values than those shown in Figure 4 for clusters in the three most remote distance categories.

Peebles and Hauser, 1974; Peebles, 1974). The sources are the Abell (1958) catalogue, the galaxies catalogued by Zwicky and his associates (Zwicky et al., 1961-68), and the galaxies catalogued from the Lick Astrographic plates (Shane and Wirtanen, 1967). Peebles defines the covariance function, $\omega(\theta)$ as follows: suppose two types of objects, $a$ and $b$, are distributed across the sky. The probability, $\delta P$, that an object of type $b$ will be found in a small randomly selected element of solid angle $\delta \Omega$ at an angular distance $\theta$ from an object of type $a$ is

$$
\delta P=N_{b}\left[1+\omega_{a b}(\theta)\right] \delta \Omega,
$$

where $N_{b}$ is the numerical density of objects of type $b$ on the sky. The mean value of $\omega_{a b}(\theta)$ is estimated by counting all objects of type $b$ at an angular distance $\theta$ from each of the source objects, $a$, and dividing by the solid angle counted over and by $N_{b}$. The covariance function should be 0 for all $\theta>0$ there is no angular correlation of the distribution of objects $a$ and $b$, whereas positive values of $\omega$ indicate that a correlation does exist. 
Peebles and Hauser (1974) have investigated the correlation between objects in the individual catalogues and the cross correlations of objects in different catalogues. Figure 6, adapted from Hauser and Peebles (1973) shows $\omega(\theta)$ vs $\theta$ for the clusters in the Abell catalogue. The symbols are means of estimates of $\omega$ made separately for four different parts of the catalogue.

Shane and Wirtanen (1954) have called attention to large inhomogeneities or clouds of galaxies and clusters on the Lick plates. Peebles and Hauser (1974) have analyzed both the Zwicky and Shane-Wirtanen galaxy distribution with the covariance function and confirm correlation over large angles, corresponding to a linear scale of the order $50 \mathrm{Mpc}$. Peebles also finds significant cross correlation between positions of Abell clusters and galaxies in the Shane-Wirtanen catalogue. Figure 7, adapted from Peebles (1974), shows the covariance function for the cross correlation between Abell distance class 4, richness class 1 clusters and the Lick galaxies. Peebles also found similar correlation between galaxy positions and the clusters of other distance classes, except for distance class 6 clusters, which are more remote than most of the galaxies at the Lick magnitude limit. Thus the clusters and individual galaxies seem to correlate in direction, both separately and with each other, over angular distances of up to $6^{\circ}$. The linear size of these inhomogeneities is of the order $100 \mathrm{Mpc}$.

Peebles has also developed a powerful statistical method for detecting variations over the surface distribution of galaxies or clusters by means of a two-dimensional power spectrum. It was first applied (Yu and Peebles, 1969) to test the hypothesis of complete second-order clustering of the Abell catalogue clusters. $\mathrm{Yu}$ and Peebles found that if second-order clusters contain an average of 10 rich clusters each, then only about $10 \%$ or less of the Abell clusters can be members of such superclusters, and that in a model of complete superclustering, on the average there could be at most

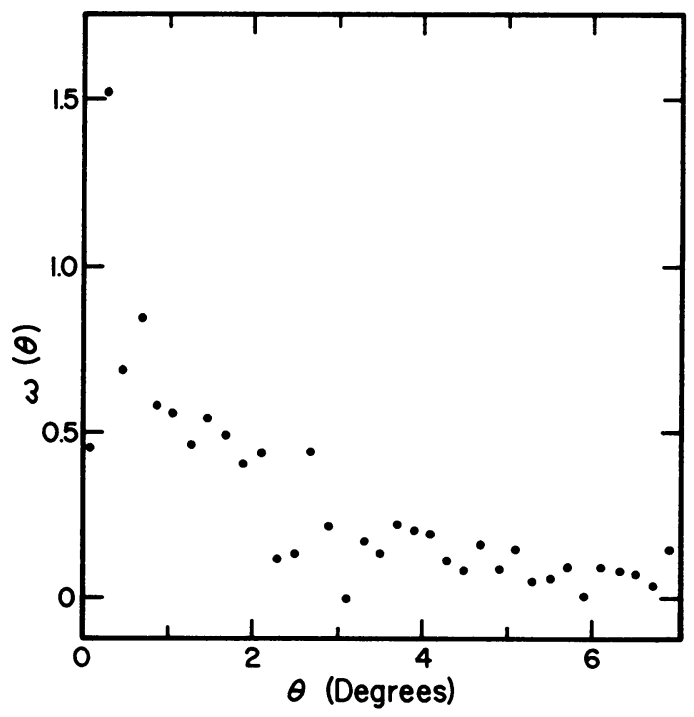

Fig. 6. The covariance function $\omega(\theta)$ vs $\theta$ for clusters in the Abell catalogue. 


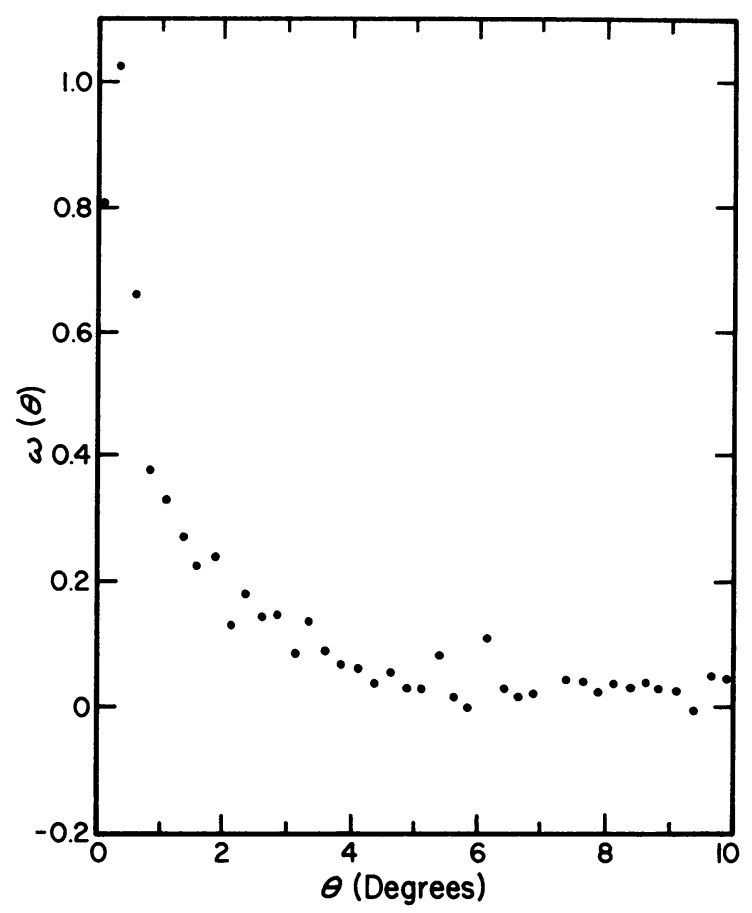

Fig. 7. The covariance function for the cross correlation between Shane-Wirtanen galaxies and clusters in the Abell Catalogue of richness class 1 and distance class 4.

about 2 clusters per supercluster. It should be noted that in these calculations those clusters of distance class 5 in the southern galactic hemisphere, where inspection of Figure 2 suggests second-order clustering to be most pronounced, were omitted because that part of the Abell catalogue seemed to $\mathrm{Yu}$ and Peebles to be atypical.

Peebles (1973b) developed the power-spectrum approach further, and he and Hauser reanalyzed the Abell catalogue (Hauser and Peebles, 1973). They report 'clear and direct evidence of superclusters with small angular scale' and that the structure corresponds to an average of 2 to 3 clusters per supercluster.

The early $\chi^{2}$ and dispersion tests described above are subject to misinterpretation because of the possibility of a general absorption gradient and other systematic effects, and the results of these tests alone should thus be viewed with caution. However, as we have seen, the same results are obtained when the covariance function is used to test for correlation between clusters, clusters and galaxies, and between galaxies. Finally, the results are again confirmed with the powerful power-spectrum analysis. We close this section by concluding that the studies of the catalogues of observed galaxies and clusters of galaxies show very strong - perhaps overwhelming evidence for inhomogeneities in the large-scale distribution of matter in space with a scale (for $H=50 \mathrm{~km} \mathrm{~s}^{-1} \mathrm{Mpc}^{-1}$ ) of the order $10^{8} \mathrm{pc}$. 


\section{Dynamical Considerations}

If a supercluster is gravitationally bound its total energy must be negative; that is

$$
T+\Omega \leqslant 0 \text {. }
$$

The kinetic energy, $T$, is $\frac{1}{2} M\left\langle V^{2}\right\rangle$, where $M$ is the total mass of the system and $\left\langle V^{2}\right\rangle$ the mean velocity dispersion, weighted by mass. The potential energy, $\Omega$, is $G M^{2} / R^{\prime}$, where $R^{\prime}$ is the mean linear separation of the members. If mass is in solar units and $\mathbf{R}^{\prime}$ in parsecs, we have

$$
\left\langle V^{2}\right\rangle^{1 / 2}=6.5 \times 10^{-2}\left(M / R^{\prime}\right)^{1 / 2} \mathrm{~km} \mathrm{~s}^{-1} .
$$

If a supercluster were in dynamical equilibrium so that the virial theorem applies, $\left\langle V^{2}\right\rangle^{1 / 2}$ would be less by only a factor of $2^{1 / 2}$.

A typical rich cluster has a mass in the range $10^{14}$ to $10^{15} M_{\odot}$ (as determined from the virial theorem, and hence including any so-called 'missing mass'). The mass of a second-order cluster should, therefore, lie in the range $10^{15}$ to $10^{17} M_{\odot}$. The total radius of a supercluster could be as large as $50 \mathrm{Mpc}$. but the known clustering within it leads to a smaller mean separation of galaxies. The appropriate value of $R^{\prime}$ should, therefore, lie in the range 10 to $50 \mathrm{Mpc}$. The extreme range of $\left\langle V^{2}\right\rangle^{1 / 2}$ to be expected is thus from about 300 to $6500 \mathrm{~km} \mathrm{~s}^{-1}$ and the value probably is in the range 1000 to $3000 \mathrm{~km} \mathrm{~s}^{-1}$. If the velocity field is isotropic, the observed rms dispersion in radial velocity would in any case be under $3000 \mathrm{~km} \mathrm{~s}^{-1}$, and probably not over $1000 \mathrm{~km} \mathrm{~s}^{-1}$ - in other words, of the same order as for an individual cluster.

Alternatively, if we considered a supercluster to be a collection of clusters, the rms velocity dispersion of clusters with respect to each other should also lie in the range 1000 to $3000 \mathrm{~km} \mathrm{~s}^{-1}$. Except for the local supercluster, for which the data are difficult to interpret, we have very little observational material with which to compare these estimates. Radial velocities are known, however, for 6 clusters that are suspected of making up a second-order cluster covering an elongated region centered near $\alpha=16^{\mathrm{h}} 14^{\mathrm{m}}, \delta=+29^{\circ}$ (Abell, 1961). The total range of these 6 velocities is about $3000 \mathrm{~km} \mathrm{~s}^{-1}$. There are not enough data to determine a meaningful velocity dispersion for the system, but at least the observations are not incompatible with the assumption that gravitational interactions occur between its members.

On the other hand, if de Vaucouleurs is correct in his suggestion that the local supercluster is expanding, and if it is typical of other superclusters, they may not have negative energy. Unless it had internal energy sources, however, an upper limit to the energy of a supercluster would be if it were expanding with the Universe. In this case, the total spread in any one component of velocity across the system would be

$$
\Delta V=H \times D=50 \times 100=5000 \mathrm{~km} \mathrm{~s}^{-1} .
$$

This, of course, would be the observed spread in radial velocity. A computed velocity dispersion would thus be of the same order as for the case of zero or negative energy. Clearly, critical observations are needed before anything meaningful can be said about the stabilities or total energies of superclusters. 
Something should be noted, however, about how superclustering might affect the dynamical studies of individual clusters. If second-order clustering is general, firstorder clusters ought not to be regarded as isolated systems, but as subcondensations within larger units. A system with subclustering has a larger (in absolute value) potential energy per unit mass than one without. The mass derived for a system from the virial theorem or from an assumption of negative total energy (the difference is only a factor of two) is proportional to the assumed value of $R^{\prime}$. Now in the analysis of a system like the Coma cluster, most investigators (e.g., Rood et al., 1972) accept as members of the cluster those galaxies in the same solid angle of the sky that have radial velocities near that of the mean for the cluster. If, however, the Coma cluster is a subcondensation in a supercluster many galaxies that meet the usual criteria for membership may not in fact be bound to the condensation itself. Even some galaxies in the direction of the cluster center can be foreground or background objects in the same supercluster. For example, there are many spiral and irregular galaxies in the fields of clusters A1656 (Coma) and A2199. The surface distributions of these galaxies, however, are quite different from those of the elliptical galaxies that dominate the membership of the main condensations of these clusters (Abell, 1974; Rood, 1974). Rood, from his analysis, concludes that some of the spirals in the field of A1656 are really cluster members, but that few, if any, of the spirals in the field of A2199 are members of that cluster.

At least three effects can result from this difficulty in identifying which galaxies should be regarded as members of a cluster under investigation:(1) the dispersion of velocities can be incorrectly estimated by including unbound galaxies in its calculation; (2) too large a radius can be attributed to the cluster; (3) an incorrect conclusion can be drawn concerning the segregation of members by mass that might result from dynamical evolution of the cluster.

Much point has been made in recent years about the high masses that are derived for some clusters of galaxies from their internal kinematics. We could imagine, however, that a cluster might have a relatively stable condensed core but be exchanging some galaxies with others in a surrounding supercluster, which in itself might have negative total energy. We have too little data for discussion of the problem now, but I suggest that any conclusions about the mass or stability of a cluster should take into account the possible effects of superclustering.

\section{Acknowledgement}

It is a pleasure to thank Dr P. J. E. Peebles for permission to reproduce some of his statistical results prior to their publication.

\section{References}

Abell, G. O.: 1957, Thesis, California Institute of Technology, Pasadena, California.

Abell, G. O.: 1958, Astrophys. J. Suppl. Ser. 3, 211.

Abell, G. O.: 1961, Astron. J. 66, 607. 
Abell, G. O.: 1974, Stars and Stellar Systems 9,

Abell, G. O. and Seligman, C. E. : 1965, Astron. J. 70, 317 (Abstract).

Abell, G. O. and Seligman, C. E. : 1967, Astron. J. 72, 288 (Abstract).

Bogart, R. S. and Wagoner, R. V.: 1973, Astrophys. J. 181, 609.

Carpenter, R. L.: 1961, Publ. Astron. Soc. Pacific 73, 324 (Abstract).

De Vaucouleurs, G. : 1953, Astron. J. 58, 30.

De Vaucouleurs, G.: 1956, Vistas in Astronomy 2, 1584-1606.

De Vaucouleurs, G.: 1958, Astron. J. 63, 253.

Hauser, M. G. and Peebles, P. J. E. : 1973, Astrophys. J. 185, 757.

Holmberg, E. : 1937, Ann. Obs. Lund. 6,

Hubble, E. P.: 1936, The Realm of the Nebulae, Oxford Univ. Press, London, pp. 72-82.

Karpowicz, M.: 1967, Z. Astrophys. 66, 301.

Kiang, T. and Saslaw, W. C.: 1969, Monthly Notices Roy. Astron. Soc. 143, 129.

Neyman, J. and Scott, E. L.: 1952, Astrophys. J. 116, 144.

Neyman, J., Scott, E. L., and Shane, C. D.: 1953, Astrophys. J. 117, 92.

Neyman, J., Scott, E. L., and Shane, C. D.: 1954, Astrophys. J. Suppl. Ser. 1, 269.

Peebles, P. J. E.: 1973a, Meeting of the Astronomical Soc. of the Pacific, Univ. of Southern Calif., June.

Peebles, P. J. E. : 1973b, Astrophys. J. 185, 413.

Peebles, P. J. E.: 1974, Astrophys. J. Suppl. Ser., in press.

Peebles, P. J. E. and Hauser, M. G.: 1974, Astrophys. J. Suppl. Ser., in press.

Reiz, A.: 1941, Ann. Obs. Lund. 9, 65.

Rood, H. J.: 1974, Publ. Astronomical Soc. of the Pacific 86, 99.

Rood, H. J., Page, T. L., Kitner, E. C., and King, I. R.: 1972, Astrophys. J. 175, 627.

Scott, E. L., Shane, C. D., and Swanson, M. D.: 1954, Astrophys. J. 119, 91.

Shane, C. D. and Wirtanen, C. A.: 1954, Astron. J. 59, 285.

Shane, C. D. and Wirtanen, C. A.: 1967, Publ. Lick Obs. 22, Part 1, p.

Yu, J. T. and Peebles, P. J. E.: 1969, Astrophys. J. 158, 103.

Zwicky, F.: 1953, Hetvet. Phys. Acta 26, 241.

Zwicky, F.: 1957, Morphological Astronomy, Springer-Verlag, Berlin, Ch. 2-5.

Zwicky, F.: 1959, Handbuch der Physik 53, 390.

Zwicky, F. and Rudnicki, K.: 1963, Astrophys. J. 137, 707.

Zwicky, F. and Rudnicki, K.: 1966, Z. Astrophys. 64, 246.

Zwicky, F., Herzog, E., Wild, P., Karpowicz, M., and Kowal, C. T. : 1961-68, Catalogue of Galaxies and Clusters of Galaxies (in 6 volumes), California Institute of Technology, Pasadena.

\section{DISCUSSION}

Scott: The space distribution of galaxies was studied in the earlier papers by Neyman, Shane and myself. We found an excellent fit to the counts of Shane and Wirtanen made in $1^{\circ} \times 1^{\circ}$ sq and also to the counts in $10^{\prime} \times 10^{\prime}$ squares but these were not consistent. Our $1^{\circ} \times 1^{\circ}$ results are consistent with those of Peebles.

Von Hoerner: You have given good evidence for general inhomogeneities on a large scale. Would you go one step further - is there evidence for distinct 'superclusters' (to be catalogued) and is there a hierarchy?

Abell: Yes, A couple of dozen or so apparent groups of rich clusters can easily be identified. I published a list and description of 17 probable superclusters in the Astronomical Journal 66, 607.

Icke: Is it possible to gauge the correctness of the statistical techniques by applying them to stars, for which the spatial distribution is reasonably well known?

Abell: Yes, the distribution of stars in grid cells in the sky has been looked at many times. In most regions, it resembles the binomial distribution expected for random, non-interacting objects. There are deviations in regions of star clustering and interstellar absorption. Serial correlation techniques have also been applied to study the distribution of absorbing matter and fluctuations in the Milky Way brightness for example by Limber and by Chandrasekhar and Münch. I am not aware that the powerful twodimensional power-spectrum technique, like that of Peebles and Hauser, has ever been applied to star distributions.

Partridge: Peebles is now working on star counts using his technique. 